\title{
Intravitreal Dexamethasone Implant in Patients Who Did Not Complete Anti-VEGF Loading Dose During the COVID-19 Pandemic: a Retrospective Observational Study
}

\author{
Vincenzo Scorcia • Giuseppe Giannaccare • Valentina Gatti · Sabrina Vaccaro • Gabriele Piccoli • \\ Annarita Villì · Mario Damiano Toro · Angeli Christy Yu • Claudio Iovino · Francesca Simonelli • \\ Adriano Carnevali (D)
}

Received: July 21, 2021 / Accepted: August 25, 2021 / Published online: September 5, 2021

(c) The Author(s) 2021

\section{ABSTRACT}

Introduction: To compare the functional and anatomic outcomes between eyes in patients with diabetic macular edema (DME) who underwent a complete anti-vascular endothelial growth factor (VEGF) loading dose with aflibercept and those who were switched to dexamethasone intravitreal (DEX) implant after an incomplete anti-VEGF treatment regimen during the coronavirus disease 2019 (COVID19) pandemic.

Methods: This was a retrospective and comparative study conducted on patients with DME. Main outcome measures were mean

V. Scorcia · G. Giannaccare · V. Gatti · S. Vaccaro · G. Piccoli · A. Villì · A. Carnevali $(\square)$

Department of Ophthalmology, University of Magna Graecia of Cantazaro, Viale Europa, Loc. Germaneto, 88100 Catanzaro, Calabria, Italy e-mail: adrianocarnevali@live.it

M. D. Toro

Department of Ophthalmology, University Hospital of Zurich, University of Zurich, 9081 Zurich, Switzerland

\section{A. C. $\mathrm{Yu}$}

Department of Translational Medicine, University of Ferrara, Ferrara, Italy

C. Iovino · F. Simonelli

Multidisciplinary Department of Medical, Surgical and Dental Sciences, Eye Clinic, University of Campania Luigi Vanvitelli School of Medicine and Surgery, Naples, Italy change in best corrected visual acuity (BCVA) and central retinal thickness (CRT) from baseline to month 4 .

Results: Forty-three eyes (23 eyes in the antiVEGF group and 20 eyes in the DEX group) were included. Mean BCVA significantly improved from $37.7 \pm 25.3$ and $35.7 \pm 22.0$ letters at baseline to 45.4 (23.9) (mean adjusted BCVA improvement $7.6 \pm 20.8$ letters, $p=0.033)$ and $46.1 \pm 26.0$ (mean adjusted BCVA improvement $10.6 \pm 15.9$ letters, $p=0.049$ ) at month 4 in the anti-VEGF and DEX groups, respectively, with no significant differences between study groups (mean adjusted BCVA difference 2.8 letters, $95 \% \mathrm{CI}-9.4$ to 14.9 letters, $p=0.648$ ). There were no statistically significant differences in the proportion of eyes that achieved a BCVA improvement of $\geq 5, \geq 10$, and $\geq 15$ letters between groups. CRT was significantly reduced from baseline to month 4 in both DEX (mean adjusted CRT reduction $167.3 \pm 148.2 \mu \mathrm{m}, p=0.012)$ and anti-VEGF groups (mean adjusted CRT reduction $109.9 \pm 181.9 \mu \mathrm{m}, p<0.001)$, with no differences between them (mean adjusted CRT difference $56.1 \mu \mathrm{m}, 95 \% \mathrm{CI}-46.0$ to $158.2 \mu \mathrm{m}$, $p=0.273)$. Of 20 eyes in the DEX group, 16 $(80.0 \%)$ and $9(45.0 \%)$ eyes achieved a CRT reduction of $\geq 20 \%$ from baseline at 2 months and at 4 months, respectively.

Conclusions: Our results seem to suggest that DEX implant can significantly improve both functional and anatomic clinical outcomes in 
patients who were unable to complete antiVEGF loading dose during the COVID-19 pandemic.

Keywords: COVID-19; Dexamethasone Intravitreal Implant; Diabetes; Diabetic Macular Edema; Diabetic Retinopathy; Vascular Endothelial Growth Factor Inhibitors

\section{Key Summary Points}

Why carry out this study?

Switching to a sustained released dexamethasone intravitreal (DEX) implant has seemed to be a feasible option in patients with diabetic macular edema who do not adequately respond to intravitreal vascular endothelial growth factor inhibitor (anti-VEGF) injections.

Measures adopted during the coronavirus disease 2019 (COVID-19) pandemic have dramatically disrupted the provision of health care, resulting in deferral of routine ophthalmic procedures.

This study aimed to compare functional and anatomic outcomes in patients with diabetic macular edema who underwent a full anti-VEGF regimen (monthly injection during 5 months) versus those who underwent dexamethasone intravitreal implant after an incomplete anti-VEGF treatment regimen.

\section{What was learned from the study?}

DEX implant significantly improved both functional and anatomic outcomes in patients who were unable to complete anti-VEGF loading dose.

DEX implant may be a valuable option for treating patients with DME who have not completed the standard anti-VEGF loading dose regimen.

\section{INTRODUCTION}

Diabetic macular edema (DME) is the leading cause of vision loss in patients with diabetes [1]. DME is a chronic, multifactorial, and sightthreatening condition that critically impacts on the patient's quality of life [2, 3]. Many different treatment options are currently available for DME; among these, intravitreal injections of anti-vascular endothelial growth factor inhibitors (VEGF) are considered the first line of treatment in center-involving DME [4]. However, on the basis of large clinical trial results, only $33-45 \%$ of patients with DME on antiVEGF agents showed three lines or more of visual improvement [4-6], which supports the presence of multiple factors contributing to the pathophysiology of DME [5-7]. Since there is increasing evidence about the role of inflammation in the pathophysiology of DME, corticosteroids have taken an active role in its treatment [7-11].

With the coronavirus disease 2019 (COVID19) outbreak over the last year, the strategies adopted by different governments to reduce the risk of infection spread have dramatically disrupted the provision of health care, resulting in deferral of routine ophthalmic procedures $[12,13]$. As a result of the risk of irreversible visual loss, treatment planning and prioritization during the pandemic has become more critical than ever in order to achieve and maintain optimal clinical outcomes in patients with DME [14, 15]. Although we have established specific schedules for patients with DME [16], many patients were unwilling to follow the recommended treatment regimen [16].

Switching from anti-VEGF injections to a sustained released dexamethasone intravitreal (DEX) implant has been shown to provide good functional and anatomic outcomes [17-20]. Thus, because we have encountered many patients with DME who were unable to comply with the anti-VEGF scheduled treatment regimen, we considered DEX implant as an option for treating these patients during the COVID-19 pandemic.

This study aimed to compare functional and anatomic outcomes in patients with DME who 
underwent a full anti-VEGF regimen (monthly injection during 5 months) versus those who underwent DEX treatment after an incomplete anti-VEGF treatment regimen.

\section{METHODS}

This retrospective comparative study included 20 patients with DME who were unable to receive a complete loading regimen of at least five consecutive anti-VEGF injections because of the COVID-19 pandemic and subsequently underwent treatment with DEX implant from April 2020 to January 2021 and 23 patients with DME treated from January 2016 to February 2020 with five monthly intravitreal injections of aflibercept as a control group, at a tertiary level hospital (University of Magna Graecia, Catanzaro, Italy). This study adhered to the tenets of the Declaration of Helsinki and was approved by the local ethics committee of the University of Magna Graecia, Catanzaro, Italy.

Inclusion criteria were age 18 years or older, diagnosis of type 2 diabetes mellitus, HbA1c $\leq 9 \%(75 \mathrm{mmol} / \mathrm{mol})$, sufficiently clear ocular media, adequate pupillary dilation, fixation to permit high quality optical coherence tomography (OCT) imaging; macular edema defined clinically and by structural spectral domain (SD)-OCT as the presence of central retinal thickness (CRT) of $\geq 250 \mu \mathrm{m}$ with intra/subretinal fluid and not having received any antiVEGF treatment before starting the study. Exclusion criteria included concomitant ocular disease that could cause macular edema including choroidal neovascularization, retinal vein occlusion, uveitis and recent intraocular surgery less 6 months prior, and the presence of any retinal disease other than diabetic retinopathy (i.e., hypertensive retinopathy, central serous retinopathy, or macular dystrophies). Both phakic and pseudophakic patients were included in the study. Minimum follow-up was 4 months.

If both eyes were eligible, only one eye (chosen at random) was used for the analysis.

Patients who had been switched from antiVEGF therapy to DEX implant for any reason other than inability to attend consultation due to COVID restrictions were excluded from the study.

\section{Assessments}

Each patient was assessed at baseline (prior to aflibercept injection for the anti-VEGF group and prior to DEX implantation for the DEX group) and 2 and 4 months after both interventions. During each follow-up visit, comprehensive ophthalmologic examination was performed including best corrected visual acuity (BCVA) testing using the standard early treatment diabetic retinopathy study (ETDRS) protocol, slit lamp examination, intraocular pressure (IOP) measurement, and dilated fundus biomicroscopy. Structural SD-OCT (Optovue; Optovue Inc., Fremont, CA, USA) was also performed at every visit. Complications were also assessed in all follow-up visits.

\section{Outcomes}

Main outcome measures were change in BCVA and CRT from baseline to month 4 .

Secondary outcome measures included incidence of adverse events and proportion of eyes that achieved a CRT reduction of $\geq 20 \%$ from baseline to month 4; proportion of eyes achieving a BCVA improvement of $\geq 5, \geq 10$, and $\geq 15$ letters; and proportion of eyes losing $\geq 5, \geq 10$, and $\geq 15$ letters in BCVA. Anatomic success was defined as a CRT reduction of $\geq 10 \%$ from baseline to month 4 .

\section{Data Analysis}

All statistical analysis was performed using IBM SPSS (version 27.0; IBM, Armonk, New York). For descriptive statistics, number (percentage), mean (standard deviation, SD), mean (95\% confidence interval, CI), and median (interquartile range) were used as appropriate.

Data were tested for normal distribution using a Shapiro-Wilk's test.

A Friedman's two-way analysis test was used to assess the changes in BCVA and CRT.

Analysis of covariance (ANCOVA) was used to compare changes in BCVA and CRT between 
study groups. The model included "group" as a factor and age, baseline BCVA, and previous laser photocoagulation as covariates. Poisson regression analysis was used to compare the proportion of patients who achieved a specific BCVA gain or loss. Categorical variables were compared using a Fisher's exact or chi-squared test as appropriate.

\section{RESULTS}

Forty-three eyes were included, 23 (53.5\%) eyes in the anti-VEGF group and 20 (46.5\%) eyes in the DEX group. Mean age was $63.6 \pm 9.3$ and $62.3 \pm 7.4$ years in the in the anti-VEGF and DEX groups, respectively $(p=0.617)$. Minimum and maximum follow-up were 115 days and 136 days for the DEX group and 113 days and 139 days for the anti-VEGF group.

Table 1 summarizes the main demographic and clinical characteristics of the study population. In the DEX group, mean number of intravitreal anti-VEGF injections was $2.4 \pm 0.7$. Time between the last anti-VEGF injection and first DEX implant in the switch group was $160.7 \pm 102.8$ days (range 91.0-218.9 days).

Mean BCVA significantly improved from $37.7 \pm 25.3$ and $35.7 \pm 22.0$ letters at baseline to 45.4 (23.9) (mean adjusted BCVA improvement $7.6 \pm 20.8$ letters, $p=0.033)$ and $46.1 \pm 26.0$ (mean adjusted BCVA improvement $10.6 \pm 15.9$ letters, $p=0.049$ ) at month 4 in the anti-VEGF and DEX groups, respectively; there were no significant differences between study groups (mean adjusted BCVA difference 2.8 letters, $95 \% \mathrm{CI}-9.4$ to 14.9 letters, $p=0.648$ ) (Fig. 1).

There were no statistically significant differences in the proportion of eyes that achieved a BCVA improvement of $\geq 5, \geq 10$, and $\geq 15$ letters between groups (Fig. 2). Similarly, there were no differences in the proportion of eyes losing $\geq 5, \geq 10$, and $\geq 15$ letters (Fig. 2).

In the DEX group, there was a statistically significant reduction in CRT from $583.6 \pm 129.4 \mu \mathrm{m}$ at baseline to $473.7 \pm 197.0 \mu \mathrm{m}$ at month 4 (mean adjusted CRT reduction $167.3 \pm 148.2 \mu \mathrm{m}, p=0.012$ ). Similarly, CRT was significantly reduced in the
anti-VEGF group from $560.1 \pm 83.7 \mu \mathrm{m}$ at baseline to $392.7 \pm 146.9 \mu \mathrm{m}$ at month 4 (mean adjusted CRT reduction $109.9 \pm 181.9 \mu \mathrm{m}$, $p<0.001)$. There were no differences between groups in mean CRT reduction (mean adjusted CRT difference $56.1 \mu \mathrm{m}, 95 \% \mathrm{CI}-46.0$ to $158.2 \mu \mathrm{m}, p=0.273$ ) (Fig. 3).

Of 20 eyes in the DEX group, $16(80.0 \%)$ and $9(45.0 \%)$ eyes achieved a CRT reduction of $\geq 20 \%$ from baseline at 2 months and at 4 months, respectively. At month 4 , anatomic success was achieved in $12(60.0 \%)$ eyes in the DEX group and $16(69.6 \%)$ in the anti-VEGF group $(p=0.515)$.

Regarding safety, 1 eye (4.3\%) developed IOP elevation in the anti-VEGF group and $3(15.0 \%)$ in the DEX group $(p=0.233)$. In all cases topical medications were effective to manage the IOP. Of the 10 phakic eyes at baseline, only $1(10.0 \%)$ eye of a 68 -year-old patient in the DEX group developed visually significant lens opacification throughout the follow-up. Additionally, 1 eye $(4.3 \%)$ in the anti-VEGF group and 2 eyes $(10.0 \%)$ in the DEX group experienced a BCVA loss of $\geq 15$ letters, $(p=0.482)$.

\section{DISCUSSION}

The results of the current paper have shown that DEX implant may be a valuable option for treating patients with DME who have not completed the standard anti-VEGF loading dose regimen.

COVID-19 has posed a global public health threat due to the severity of its contagion and associated morbidity and mortality $[16,17]$. In fact, the volume of outpatient and surgical procedures has significantly decreased during the pandemic [12]. In most ophthalmic centers, provision of care was restricted to urgent or emergency cases [21].

Anti-VEGF intravitreal injections are currently considered the standard of care for patients with DME [4]. However, since unintentional treatment interruptions can result in severe adverse visual sequelae $[22,23]$, evaluation of alternative longer-term therapy is crucial for achieving optimal clinical outcomes among patients with DME [14]. The positive effect of 
Table 1 Baseline demographic and clinical characteristics

\begin{tabular}{|c|c|c|c|}
\hline & Anti-VEGF group $(n=23)$ & DEX group $(n=20)$ & $p$ value $^{a}$ \\
\hline \multicolumn{4}{|l|}{ Age, years } \\
\hline Mean (SD) & $63.6(9.3)$ & $62.3(7.4)$ & $0.617^{\mathrm{c}}$ \\
\hline \multicolumn{4}{|l|}{ Sex, $n(\%)$} \\
\hline Women & $5(21.7)$ & $7(35.0)$ & 0.497 \\
\hline Men & $18(78.3)$ & $13(65.0)$ & \\
\hline \multicolumn{4}{|c|}{ Number of IVI*, $n(\%)$} \\
\hline 1 & $0(0.0)$ & $2(10.0)$ & $<0.001^{\mathrm{b}}$ \\
\hline 2 & $0(0.0)$ & $9(45.0)$ & \\
\hline 3 & $0(0.0)$ & $9(45.0)$ & \\
\hline 5 & $23(100.0)$ & $0(0.0)$ & \\
\hline \multicolumn{4}{|c|}{ Lens status, $n(\%)$} \\
\hline Phakic & $4(17.4)$ & $6(30.0)$ & 0.473 \\
\hline Pseudophakic & $19(82.6)$ & $14(70.0)$ & \\
\hline \multicolumn{4}{|c|}{ Preoperative laser, $n(\%)$} \\
\hline None & $13(56.5)$ & $8(40.0)$ & $0.478^{\mathrm{b}}$ \\
\hline PRP & $4(17.4)$ & $6(30.0)$ & \\
\hline Macular grid & $0(0.0)$ & $1(5.0)$ & \\
\hline Combined $^{* *}$ & $6(26.1)$ & $5(25.0)$ & \\
\hline \multicolumn{4}{|c|}{ Presence of IRF, $n(\%)$} \\
\hline No & $6(26.1)$ & $4(20.0)$ & 0.728 \\
\hline Yes & $17(73.9)$ & $16(80.0)$ & \\
\hline \multicolumn{4}{|l|}{ Presence of SRF } \\
\hline No & $22(95.7)$ & $16(80.0)$ & 0.167 \\
\hline Yes & $1(4.3)$ & $4(20.0)$ & \\
\hline \multicolumn{4}{|l|}{ PDR, $n(\%)$} \\
\hline Yes & $14(60.9)$ & $12(60.0)$ & 1.000 \\
\hline No & $9(39.1)$ & $8(40.0)$ & \\
\hline \multicolumn{4}{|l|}{$\mathrm{BCVA}^{* * *}$} \\
\hline Mean (SD) & $37.7(25.3)$ & $35.7(22.0)$ & $0.785^{\mathrm{c}}$ \\
\hline CRT & & & \\
\hline
\end{tabular}


Table 1 continued

\begin{tabular}{lllr}
\hline & Anti-VEGF group $(\boldsymbol{n}=\mathbf{2 3})$ & DEX group $(\boldsymbol{n}=\mathbf{2 0})$ & $\boldsymbol{p}_{\text {value }}$ \\
\hline Mean $(\mathrm{SD})$ & $560.1(83.7)$ & $583.6(129.4)$ & $0.478^{\mathrm{c}}$ \\
\hline
\end{tabular}

$V E G F$ vascular endothelial grow factor, $D E X$ intravitreal dexamethasone implant, $S D$ standard deviation, $I V I$ intravitreal injections, $P R P$ pan retinal photocoagulation, $I R F$ intraretinal fluid, $S R F$ subretinal fluid, $P D R$ proliferative diabetic retinopathy, BCVA best corrected visual acuity, $C R T$ central retinal thickness

${ }^{*}$ Aflibercept

${ }^{* *} \mathrm{PRP}+$ macular grid

${ }^{* * *}$ Letters in the Early Treatment Diabetic Retinopathy Study (ETDRS) charts

${ }^{a}$ Fisher exact test

${ }^{\mathrm{b}}$ Chi-squared test

c $T$ test

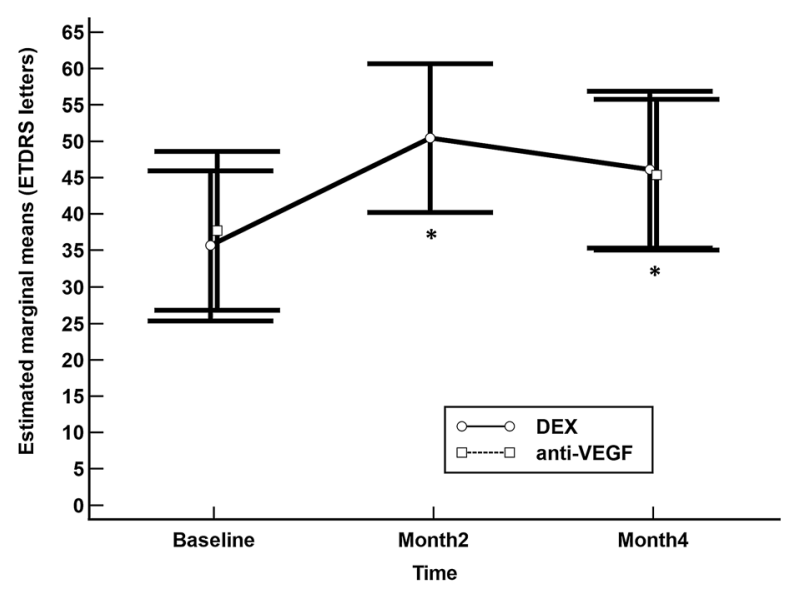

Fig. 1 Mean change in best corrected visual acuity in the anti-VEGF and dexamethasone intravitreal (DEX) implant study groups. The vertical bars represent the 95\% confidence interval. Intra-group statistical significance was determined using the Wilcoxon test. Statistical significance between groups was determined using the Mann-Whitney $U$ test. No significant differences were found between groups. ${ }^{*} p<0.05$ in both groups as compared to baseline

switching to DEX implant in eyes with persistent DME unresponsive to anti-VEGF therapy has been previously reported [17-20]. Switching to DEX implant has also been shown to result in better anatomical outcomes over those of continued anti-VEGF therapy [24-26]. In the current study, the indication for switching was not due to lack of efficacy, but rather the patient's inability to comply with a routine monthly anti-VEGF therapy.

Although, when comparing to those eyes that underwent the standard anti-VEGF therapeutic regimen, switching to DEX implant did not lead better BCVA improvement or CRT reduction, it is noteworthy that one DEX implant significantly improved both functional and anatomic clinical outcomes in those eyes that did not complete anti-VEGF loading dose.

In the DEX group median BCVA improvement between pre- and post-anti-VEGF therapy was not statistically significant (Hodges-Lehmann median difference 5.0 letters, $95 \% \mathrm{CI}$ -0.6 to 9.5 letters, $p=0.097)$. Nevertheless, there was significant reduction in CRT after anti-VEGF injections (Hodges-Lehmann median difference $117.3 \mu \mathrm{m}, 95 \% \mathrm{CI} \quad 44.0$ to $191.0 \mu \mathrm{m}, p=0.002$ ).

Moreover, after adjustment for age, previous laser, and baseline BCVA, mean BCVA improvement was greater in those eyes that were switched to DEX than in those who underwent anti-VEGF loading dose (mean difference 1.99 letters, $95 \% \mathrm{CI}-8.85$ to 12.83 ), although such a difference was not statistically significant $(p=0.712)$. However, the power to detect significant differences with the observed values was 7\%. Similarly, after adjustment for age, previous laser, and baseline CRT, there were no significant differences in mean CRT 


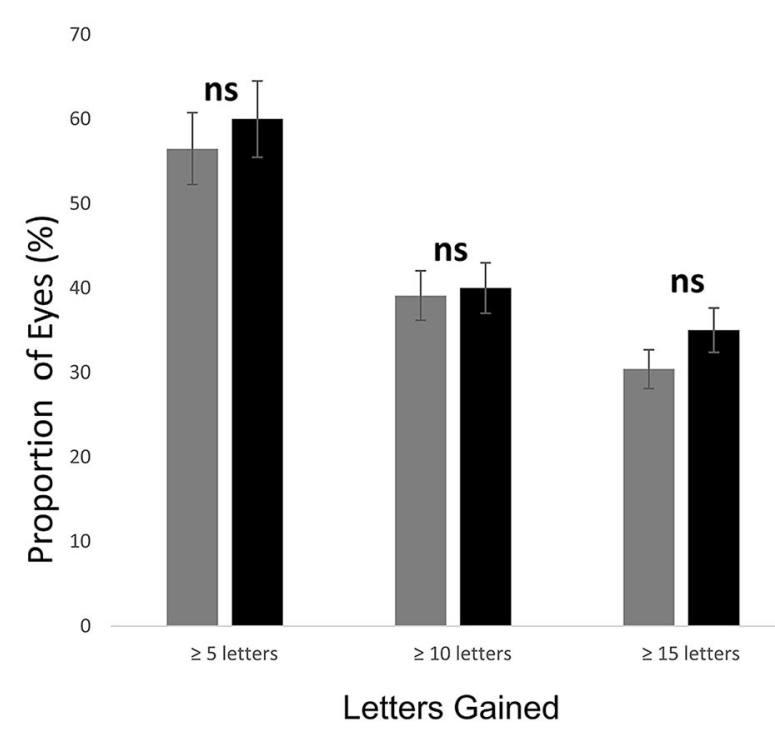

Fig. 2 Comparison in the proportion of eyes achieving a best corrected visual acuity (BCVA) improvement of $\geq 5$, $\geq 10$, and $\geq 15$ letters and a BCVA loss of $\geq 5, \geq 10$,

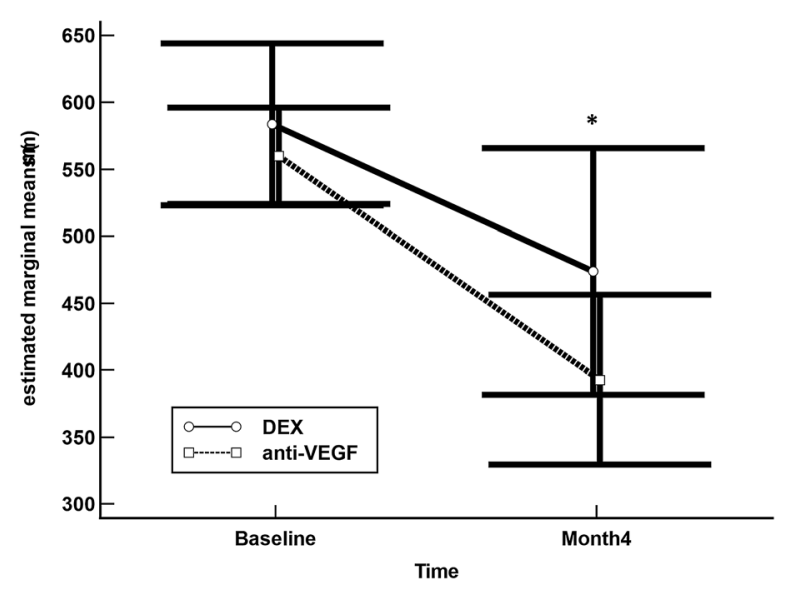

Fig. 3 Mean change in best corrected visual acuity in the anti-VEGF and dexamethasone intravitreal (DEX) implant study groups. The vertical bars represent the 95\% confidence interval. Intra-group statistical significance was determined using the Wilcoxon test. Statistical significance between groups was determined using the Mann-Whitney $U$ test. No significant differences were found between groups. ${ }^{*} p<0.05$ in both groups as compared to baseline

reduction between DEX and anti-VEGF groups (mean difference $62.0,95 \% \mathrm{CI}-39.6$ to 163.6 , $p=0.224$ ); however, as happened with BCVA, and $\geq 15$ letters in the dexamethasone intravitreal (DEX) implant and anti-VEGF groups. ns No significant difference

our study was underpowered to detect such a difference (24\%).

Regarding safety, there were no unexpected adverse events and there were no differences between groups in either incidence of ocular hypertension or cataract/lens opacification or loss of BCVA.

According to the European Society of Retina Specialists (EURETINA) guidelines [4], antiVEGF treatment should be initiated early on with monthly injections, and in those patients who experience positive anatomic and/or functional outcomes, continuing with monthly injections until visual acuity and/or OCT stability is reached. This raises the question of whether anti-VEGF treatment can be continued or should it be restarted? In any case, this would require monthly visits, which during the pandemic would not be possible. Therefore, having a therapeutic alternative, like DEX implant, which provides good anatomical and functional outcomes in both treatment naïve and antiVEGF refractory patients with DME may be considered as a valuable alternative [12, 20, 27].

The most important limitations of the current study are its retrospective design and small sample size. Selection bias and confounding factors are inherent to retrospective studies; 
nevertheless, strict inclusion/exclusion criteria aimed to minimized such issues. Additionally, our small sample size also limits the power of the analysis. In fact, with the observed pooled standard deviation and the number of eyes included in each group, the power to detect a mean BCVA improvement of 5 ETDRS letters or a CRT reduction of $25 \mu \mathrm{m}$ between DEX and anti-VEGF groups, with an alpha error of 0.05 in a bilateral contrast, would be $14 \%$ and $8 \%$, respectively. Therefore, further investigations with a larger number of recruited patients would be needed. It should be mentioned that our study did not evaluate the role of OCT biomarkers, which may provide valuable information about the predictive capacity of DEX response. Different OCT biomarkers, such as disorganization of retinal inner layers [28] or ellipsoid zone integrity $[29,30]$, may predict anatomic and functional outcomes after treatment with DEX implants. This information may be extremely useful, particularly in these times, when the pandemic limits our consultation capacity and it is crucial for optimizing our resources.

\section{CONCLUSIONS}

Despite these limitations, the results of our study suggested that DEX implant significantly improved both anatomic and functional outcomes in patients who were unable to receive a complete anti-VEGF therapeutic regime during the COVID-19 pandemic.

\section{ACKNOWLEDGEMENTS}

Funding. The authors wish to acknowledge Allergan, an AbbVie company, for their support with the medical writing and publication fees, including the journal's Rapid Service Fees. It should be noted that Allergan was not involved in the preparation of the manuscript nor did the company influence in any way the scientific conclusions reached.
Editorial Assistance. Editorial assistance in the preparation of this article was provided by Antonio Martinez (MD) of Ciencia y Deporte Ltd. Support for this assistance was funded by Allergan, an AbbVie company.

Authorship. All named authors meet the International Committee of Medical Journal Editors (ICMJE) criteria for authorship for this article, take responsibility for the integrity of the work as a whole, and have given their approval for this version to be published.

Authorship Contributions. Vincenzo Scorcia, Giuseppe Giannaccare, Valentina Gatti, Sabrina Vaccaro, Gabriele Piccoli, Annarita Villi, Mario Damiano Toro, Angeli Christy Yu, Iovino Claudio, Francesca Simonelli, and Adriano Carnevali all made substantial contributions to conception, design, analysis and interpretation of data, contributed to writing the article, provided critical revision of the manuscript, and approved the final version.

Disclosures. Vincenzo Scorcia, Giuseppe Giannaccare, Valentina Gatti, Sabrina Vaccaro, Gabriele Piccoli, Annarita Villi, Mario Damiano Toro, Angeli Christy $\mathrm{Yu}$, Iovino Claudio, and Francesca Simonelli have nothing to declare.

Dr Adriano Carnevali has received a grant from Allergan during the conduct of the study.

Neither honoraria nor payments were made for authorship of this article.

All authors declare no proprietary interest.

Compliance with Ethics Guidelines. All procedures performed in studies involving human participants were in accordance with the ethical standards of the institutional and/or national research committee and with the 1964 Helsinki declaration and its later amendments or comparable ethical standards.

Data Availability. The datasets generated during and/or analyzed during the current study are available from the corresponding author on reasonable request.

Informed Consent. The study protocol was approved by the REGIONE CALABRIA 
COMITATO ETICO SEZIONE AREA CENTRO (Protocol. n. 281 del 17 Settembre 2020), which waived the need for Informed consent.

Open Access. This article is licensed under a Creative Commons Attribution-NonCommercial 4.0 International License, which permits any non-commercial use, sharing, adaptation, distribution and reproduction in any medium or format, as long as you give appropriate credit to the original author(s) and the source, provide a link to the Creative Commons licence, and indicate if changes were made. The images or other third party material in this article are included in the article's Creative Commons licence, unless indicated otherwise in a credit line to the material. If material is not included in the article's Creative Commons licence and your intended use is not permitted by statutory regulation or exceeds the permitted use, you will need to obtain permission directly from the copyright holder. To view a copy of this licence, visit http://creativecommons.org/licenses/by$\mathrm{nc} / 4.0 /$.

\section{REFERENCES}

1. Li JQ, Welchowski T, Schmid M, et al. Prevalence, incidence and future projection of diabetic eye disease in Europe: a systematic review and metaanalysis. Eur J Epidemiol. 2020;35(1):11-23.

2. Kocur I, Resnikoff S. Visual impairment and blindness in Europe and their prevention. Br J Ophthalmol. 2002;86(7):716-22.

3. Cunha-Vaz J, Coscas G. Diagnosis of macular edema. Ophthalmologica. 2010;224(Suppl 1):2-7.

4. Schmidt-Erfurth U, Garcia-Arumi J, Bandello F, et al. Guidelines for the management of diabetic macular edema by the European Society of Retina Specialists (EURETINA). Ophthalmologica. 2017;237(4):185-222.

5. Browning DJ, Stewart MW, Lee C. Diabetic macular edema: evidence-based management. Indian J Ophthalmol. 2018;66(12):1736-50.

6. Urias EA, Urias GA, Monickaraj F, et al. Novel therapeutic targets in diabetic macular edema: beyond VEGF. Vis Res. 2017;139:221-7.
7. Das A, McGuire PG, Rangasamy S. Diabetic macular edema: pathophysiology and novel therapeutic targets. Ophthalmology. 2015;122(7):1375-94.

8. Romero-Aroca P, Baget-Bernaldiz M, Pareja-Rios A, et al. Diabetic macular edema pathophysiology: vasogenic versus inflammatory. J Diabetes Res. 2016;2016:2156273.

9. Tang J, Kern TS. Inflammation in diabetic retinopathy. Prog Retin Eye Res. 2011;30:343-58.

10. Sohn HJ, Han DH, Kim IT, et al. Changes in aqueous concentrations of various cytokines after intravitreal triamcinolone versus bevacizumab for diabetic macular edema. Am J Ophthalmol. 2011;152(4):686-94.

11. Brooks HL Jr, Caballero S Jr, Newell CK, et al. Vitreous levels of vascular endothelial growth factor and stromal-derived factor 1 in patients with diabetic retinopathy and cystoid macular edema before and after intraocular injection of triamcinolone. Arch Ophthalmol. 2004;122(12):1801-7.

12. Iovino C, Mastropasqua R, Lupidi M, et al. Intravitreal dexamethasone implant as a sustained release drug delivery device for the treatment of ocular diseases: a comprehensive review of the literature. Pharmaceutics. 2020;12(8):703.

13. $\mathrm{Wu} \mathrm{Z}$, McGoogan JM. Characteristics of and important lessons from the coronavirus disease 2019 (COVID-19) outbreak in China: summary of a report of 72314 cases from the Chinese Center for Disease Control and Prevention. JAMA. 2020;323(13):1239-42.

14. Wykoff CC, Clark WL, Nielsen JS, et al. Optimizing anti-VEGF treatment outcomes for patients with neovascular age-related macular degeneration. J Manag Care Spec Pharm. 2018;24(2):3-15.

15. EURETINA: Working Group on Medical Retina (WG-MR) of the Netherlands Ophthalmological Society (NOG). https://www.euretina.org/covid-19/ reinier-schlingemann-working-group-on-medicalretina-wg-mr-of-the-netherlands-ophthalmologicalsociety-nog/. Accessed 16 Jul 2021.

16. Carnevali A, Giannaccare G, Gatti V, et al. Intravitreal injections during COVID-19 outbreak: realworld experience from an Italian tertiary referral center. Eur J Ophthalmol. 2021;31(1):10-2.

17. Busch C, Zur D, Fraser-Bell S, et al. International Retina Group. Shall we stay, or shall we switch? Continued anti-VEGF therapy versus early switch to dexamethasone implant in refractory diabetic macular edema. Acta Diabetol. 2018;55(8):789-96. 
18. Hernández Martínez A, Pereira Delgado E, Silva Silva G, et al. Early versus late switch: how long should we extend the anti-vascular endothelial growth factor therapy in unresponsive diabetic macular edema patients? Eur J Ophthalmol. 2020;30(5):1091-8.

19. Ruiz-Medrano J, Rodríguez-Leor R, Almazán E, et al. Results of dexamethasone intravitreal implant (Ozurdex) in diabetic macular edema patients: early versus late switch. Eur J Ophthalmol. 2020;3: 1120672120929960 . https://doi.org/10.1177/ 1120672120929960.

20. Iovino C, Peiretti E, Giannaccare G, et al. Evolving treatment paradigm in the management of diabetic macular edema in the era of COVID-19. Front Pharmacol. https://doi.org/10.3389/fphar.2021. 670468.

21. Wong TY, Bandello F. Academic ophthalmology during and after the COVID-19 pandemic. Ophthalmology. 2020;127(8):e51-2.

22. Obeid A, Su D, Patel SN, et al. Outcomes of eyes lost to follow-up with proliferative diabetic retinopathy that received panretinal photocoagulation versus intravitreal anti-vascular endothelial growth factor. Ophthalmology. 2019;126(3):407-13.

23. Yang KB, Feng H, Zhang H. Effects of the COVID-19 pandemic on anti-vascular endothelial growth factor treatment in China. Front Med (Lausanne). 2020;14(7): 576275. https://doi.org/10.3389/fmed. 2020.576275 .

24. Maturi RK, Bleau L, Saunders J, et al. A 12-month, single-masked, randomized controlled study of eyes with persistent diabetic macular edema after multiple anti-VEGF injections to assess the efficacy of the dexamethasone-delayed delivery system as an adjunct to bevacizumab compared with continued bevacizumab monotherapy. Retina. 2015;35(8): 1604-14.

25. Shah SU, Harless A, Bleau L, et al. Prospective randomized subject-masked study of intravitreal bevacizumab monotherapy versus dexamethasone implant monotherapy in the treatment of persistent diabetic macular edema. Retina. 2016;36(10): 1986-1896.

26. Maturi RK, Glassman AR, Liu D, et al. Diabetic Retinopathy Clinical Research Network. Effect of adding dexamethasone to continued ranibizumab treatment in patients with persistent diabetic macular edema: a DRCR network phase 2 randomized clinical trial. JAMA Ophthalmol. 2018;136(1): 29-38.

27. Iglicki M, Busch C, Zur D, et al. Dexamethasone implant for diabetic macular edema in naive compared with refractory eyes: the International Retina Group real-life 24-month multicenter study. The IRGREL-DEX Study. Retina. 2019;39(1):44-51.

28. Zur D, Iglicki M, Sala-Puigdollers A, et al. International Retina Group (IRG). Disorganization of retinal inner layers as a biomarker in patients with diabetic macular oedema treated with dexamethasone implant. Acta Ophthalmol. 2020;98(2): 217-23.

29. Zur D, Iglicki M, Busch C, et al. International Retina Group. OCT biomarkers as functional outcome predictors in diabetic macular edema treated with dexamethasone implant. Ophthalmology. 2018;125(2):267-75.

30. Castro-Navarro V, Monferrer-Adsuara C, NavarroPalop C, et al. Effect of dexamethasone intravitreal implant on visual acuity and foveal photoreceptor integrity in macular edema secondary to retinal vascular disease. Ophthalmologica. 2021;244(1): 83-92. 